\title{
Response function of turbulence computed via fluctuation-response relation of a Langevin system with vanishing noise
}

\author{
Takeshi Matsumoto,, , Michio Otsuki, ${ }^{2}$ Ooshida Takeshi, ${ }^{3}$ Susumu Goto, ${ }^{4}$ and Akio Nakahara ${ }^{5}$ \\ ${ }^{1}$ Division of Physics and Astronomy, Graduate School of Science, Kyoto University, Kyoto, 606-8502, Japan \\ ${ }^{2}$ Department of Materials Science, Shimane University, Matsue, 690-8504, Japan \\ ${ }^{3}$ Department of Mechanical and Aerospace Engineering, Tottori University, Tottori, 680-8552, Japan \\ ${ }^{4}$ Graduate School of Engineering Science, Osaka University, Toyonaka, 560-8531, Japan \\ ${ }^{5}$ Laboratory of Physics, College of Science and Technology, Nihon University, Funabashi, 274-8501, Japan
}

(Dated: May 26, 2018)

\begin{abstract}
For a shell model of the fully developed turbulence and the incompressible Navier-Stokes equations in the Fourier space, when a Gaussian white noise is artificially added to the equation of each mode, an expression of the mean linear response function in terms of the velocity correlation functions is derived by applying the method developed for nonequilibrium Langevin systems [Harada and Sasa, Phys. Rev. Lett. 95, 130602 (2005)]. We verify numerically for the shell model case that the derived expression of the response function, as the noise tends to zero, converges to the response function of the noiseless shell model.
\end{abstract}

Introduction Tools of statistical mechanics are indispensable for research of fluid turbulence. We here focus on relation between the linear response function and the correlation function, known as fluctuation-response relation (FRR). The simplest form of the FRR is realized in thermally equilibrium systems: the linear response function is proportional to the autocorrelation function of a dynamical variable, with the proportional constant being the inverse temperature. This classical FRR does not hold in general for a driven dissipative system with a non-Gaussian distribution function in a nonequilibrium steady state as reviewed in [1]. For statistically steadystate, homogeneous and isotropic turbulence, researchers have asked the questions: (1) what kind of FRR holds? (2) can its linear response function be expressed in terms of velocity correlation functions?

The FRR of turbulence was studied first by Kraichnan in his influential closure approximation, known as the direct-interaction approximation (DIA) 2] (see also [3] ). A major goal of statistical theories is to derive the Kolmogorov energy spectrum $E(k) \propto k^{-5 / 3}$ from the incompressible Navier-Stokes equations [4, 5]. The energy spectrum $E(k)$ is the average of the equal-time autocorrelation function of the velocity Fourier modes on the spherical surface with radius $k$ in the wavenumber space. To obtain a closure for $E(k)$, Kraichnan considered the mean linear response function in DIA. In the latest versions of DIA in the Lagrangian frame of reference [6, 7], successfully reproducing the $k^{-5 / 3}$ spectrum, the autocorrelation function and the response function are proportional as a result of the closure approximation. A direct numerical simulation result, though available for the Eulerian frame only, indicates that the correlation function and the response function are not proportional at the Kolmogorov dissipation scale with moderate Reynolds numbers [8]. For a dynamical system model of turbu-

*Electronic address: takeshi@kyoryu.scphys.kyoto-u.ac.jp lence in the Lagrangian frame, known as the GledzerOhkitani-Yamada (GOY) shell model [9 11], the FRR is numerically studied in [12], demonstrating that the proportionality does not hold for the shell variables in the inertial range. This is consistent with the strong nonGaussianity of the shell variables. We here consider expressions of the response functions of the last two cases in a unified manner using a recent result of nonequilibrium statistical mechanics. The result we rely on is established by Harada and Sasa [13, 14] to derive a general FRR for a class of nonlinear Langevin systems, which has been verified experimentally in a thermally activated system since 15] (for FRR in a deterministic setting, see, e.g., [16 18]).

Obviously the macroscopic fluid dynamical system describing turbulence, where the thermal driving is unnecessary, is different from the nonlinear Langevin system. Nevertheless there appears to be a simple way to bridge the two systems: we formally add the Gaussian white noise to the fluid dynamical equations without worrying about its physical origin; next we derive various relations with the powerful weaponry of the stochastic systems [19]; finally we consider the zero limit of the noise, hoping that the relations survive, which is an approach similar to, e.g., [20 22]. Although this limit can be difficult to study theoretically, the derived relations can be studied numerically to check whether or not, with sufficiently small noise, they are good approximations for the noiseless original system.

More specifically, by adapting the method in [13, 14], we here derive the FRR of a randomly perturbed GOY shell model and the FRR of the velocity Fourier modes in the Eulerian frame of the randomly perturbed incompressible Navier-Stokes equations. We take the following steps: (i) we add Gaussian white noise to the equation of each shell variable and each velocity Fourier mode; (ii) adapting the Harada-Sasa argument, we derive formally the FRR for these randomly perturbed shell variables and the velocity Fourier modes; (iii) we consider numerically how small the noise should be so that the randomly per- 
turbed system recovers the noiseless system; (iv) we numerically check whether or not the FRR derived in (ii) is consistent with the linear response function of the noiseless system with the sufficiently small noise. Concerning (iii) and (iv) above, the numerical analysis is carried out only for the shell model case in this paper.

Derivation of the FRR We consider a version of the shell model [23], whose variables $u_{j}(t)(j=0, \ldots, N)$ are complex numbers. They are representatives of the velocity Fourier modes of the incompressible Navier-Stokes equations in the spherical shell $k_{j} \leq|\boldsymbol{k}|<k_{j+1}$ of the wavenumber space, where $k_{j}=k_{0} 2^{j}$. The equation of $u_{j}(t)$ with the complex-number Gaussian white noise $\xi_{j}(t)$ is

$$
\frac{d}{d t} u_{j}(t)=\Lambda_{j}(t)-\nu k_{j}^{2} u_{j}(t)+\xi_{j}(t)+f_{j}^{(p)}(t),
$$

where $\Lambda_{j}(t)$ includes the nonlinear term and the deterministic large-scale forcing $F_{j}(t)$ to keep the system statistically steady: $\Lambda_{j}(t)=i\left(k_{j} u_{j+2}(t) u_{j+1}^{*}(t)-\right.$ $\left.\frac{1}{2} k_{j-1} u_{j+1}(t) u_{j-1}^{*}(t)+\frac{1}{2} k_{j-2} u_{j-1}(t) u_{j-2}(t)\right)+F_{j}(t)$. Here ${ }^{*}$ denotes the complex conjugation. In Eq. (11), $\nu$ models the kinematic viscosity and the noise $\xi_{j}(t)$ has the mean and covariance

$$
\left\langle\xi_{j}(t)\right\rangle=0, \quad\left\langle\xi_{j}(t) \xi_{l}^{*}(s)\right\rangle=2 \sigma_{j}^{2} T \delta_{j l} \delta(t-s),
$$

where $T$ is the strength of the noise which we here call "temperature". We later compare numerically the FRR for small $T$ with that of the shell model without the noise. The last term of Eq. (11), $f_{j}^{(p)}(t)$, is the infinitesimal probe force by which we define the linear response function.

To obtain an expression of the linear response function, we follow the Onsager-Machlup path-integral approach [14]. The starting point is the probability functional of the Brownian paths $\xi_{j}(t)(j=0, \ldots, N)$ from time $t_{0}$ to $t$, $P\left(\boldsymbol{\xi}, t \mid \boldsymbol{\xi}_{0}, t_{0}\right)=\int_{\left(\boldsymbol{\xi}_{0}, t_{0}\right)}^{(\boldsymbol{\xi}, t)} D[\boldsymbol{\xi}] \exp \left[-\frac{1}{2} \sum_{j=0}^{N} \int_{t_{0}}^{t} \frac{\left|\xi_{j}(s)\right|^{2}}{\sigma_{j}^{2} T} d s\right]$. Change of variables from $\boldsymbol{\xi}=\left(\xi_{0}, \ldots, \xi_{N}\right)$ to $\boldsymbol{u}=$ $\left(u_{0}, \ldots, u_{N}\right)$ yields the path-integral representation of the transition probability as

$$
\begin{aligned}
& P\left(\boldsymbol{u}, t \mid \boldsymbol{u}_{0}, t_{0}\right)=\int_{\left(\boldsymbol{u}_{0}, t_{0}\right)}^{(\boldsymbol{u}, t)} D[\boldsymbol{u}] \exp \left\{-\frac{1}{2} \sum_{l=0}^{N} \int_{t_{0}}^{t} d s\right. \\
& {\left[\frac{1}{\sigma_{l}^{2} T}\left|\dot{u}_{l}(s)-\Lambda_{l}(s)+\nu k_{l}^{2} u_{l}(s)-f_{l}^{(p)}(s)\right|^{2}\right.} \\
& \left.\left.+\frac{\partial}{\partial u_{l}}\left(\Lambda_{l}(s)-\nu k_{l}^{2} u_{l}(s)+f_{l}^{(p)}(s)\right)\right]\right\} .
\end{aligned}
$$

The last divergence term can be interpreted as contribution from the Jacobian [24]. By linearizing Eq. (3) in regard to $f_{l}^{(p)}$, we obtain an expression of the ensemble average, $\left\langle u_{j}(t)\right\rangle_{p}$. The mean linear response function $G_{j l}^{(T)}$ can be then written as

$$
\begin{aligned}
G_{j l}^{(T)}(t-s)= & \frac{\delta\left\langle u_{j}(t)\right\rangle_{p}}{\delta f_{l}^{(p)}(s)}=\frac{1}{2 \sigma_{l}^{2} T}\left[\left\langle\dot{u}_{l}^{*}(s) u_{j}(t)\right\rangle\right. \\
& \left.-\left\langle\Lambda_{l}^{*}(s) u_{j}(t)\right\rangle+\nu k_{l}^{2}\left\langle u_{l}^{*}(s) u_{j}(t)\right\rangle\right] .
\end{aligned}
$$

We denote the most right-hand side of Eq. (4) by $H_{j l}^{(T)}(t-$ $s)$. Here $\langle\cdot\rangle$ represents the ensemble average taken in the absence of the probe force. For the diagonal part $G_{j j}^{(T)}$, we can simplify the expression by using the causality of the response function and the temporal symmetry of the autocorrelation function, as

$$
\begin{aligned}
G_{j j}^{(T)}(t-s)= & \frac{1}{\sigma_{j}^{2} T}\left\{\nu k_{j}^{2} C_{j j}^{(T)}(t-s)\right. \\
& \left.-\frac{1}{2}\left[\left\langle\Lambda_{j}^{*}(t) u_{j}(s)\right\rangle+\left\langle\Lambda_{j}^{*}(s) u_{j}(t)\right\rangle\right]\right\},
\end{aligned}
$$

where $C_{j j}^{(T)}(t-s)=\left\langle u_{j}(t) u_{j}^{*}(s)\right\rangle$ is the autocorrelation function. Equation (5) is the main FRR result of this paper, which we study numerically below.

It is straightforward to extend the above argument to the case of the three-dimensional incompressible Navier-Stokes equations in a periodic cube, which are written in terms of the velocity Fourier coefficients, $\quad\left(\hat{u}_{1}(\boldsymbol{k}, t), \hat{u}_{2}(\boldsymbol{k}, t), \hat{u}_{3}(\boldsymbol{k}, t)\right), \quad$ as $\frac{d}{d t} \hat{u}_{a}(\boldsymbol{k}, t)=$ $-i \sum_{b, c=1}^{3} k_{b}\left(\delta_{a c}-\frac{k_{a} k_{c}}{k^{2}}\right) \sum_{\boldsymbol{p}+\boldsymbol{q}=\boldsymbol{k}}^{\boldsymbol{p}, \boldsymbol{q}} \hat{u}_{b}(\boldsymbol{p}, t) \hat{u}_{c}(\boldsymbol{q}, t)+$ $\hat{F}_{a}(\boldsymbol{k}, t)-\nu k^{2} \hat{u}_{a}(\boldsymbol{k}, t)$. Here $k=|\boldsymbol{k}|$ and we assume that the deterministic large-scale forcing $\hat{F}_{a}$ is solenoidal, and that the number of the Fourier coefficients is finite. Due to the incompressibility $\boldsymbol{k} \cdot \hat{\boldsymbol{u}}(\boldsymbol{k}, t)=0$, $\hat{\boldsymbol{u}}(\boldsymbol{k}, t)$ has only two independent components, which we express as $\hat{\boldsymbol{u}}(\boldsymbol{k}, t)=\hat{u}_{\varphi}(\boldsymbol{k}, t) \boldsymbol{e}_{\varphi}+\hat{u}_{\theta}(\boldsymbol{k}, t) \boldsymbol{e}_{\theta}$ [26]. To the equations of the two components, we add the probe force $\left(f_{\varphi}^{(p)}, f_{\theta}^{(p)}\right)$ and the Langevin noise $\left(\xi_{\varphi}, \xi_{\theta}\right)$ satisfying $\left\langle\xi_{\alpha}(\boldsymbol{k}, t) \xi_{\beta}(\boldsymbol{q}, s)\right\rangle=2 \sigma^{2}(k) T \delta_{\alpha \beta} \delta_{\boldsymbol{k},-\boldsymbol{q}} \delta(t-s)$ with the indices $\alpha, \beta=\varphi, \theta$. The mean linear response function in the Navier-Stokes case is expressed as

$$
\begin{aligned}
G_{\alpha \beta}^{(T)}(\boldsymbol{k}, t \mid \boldsymbol{q}, s) & =\frac{\delta\left\langle\hat{u}_{\alpha}(\boldsymbol{k}, t)\right\rangle_{p}}{\delta f_{\beta}^{(p)}(\boldsymbol{q}, s)}=\frac{1}{2 \sigma(k)^{2} T}\left[\left\langle\dot{\hat{u}}_{\beta}^{*}(\boldsymbol{q}, s) \hat{u}_{\alpha}(\boldsymbol{k}, t)\right\rangle\right. \\
& \left.-\left\langle\Lambda_{\beta}^{*}(\boldsymbol{q}, s) \hat{u}_{\alpha}(\boldsymbol{k}, t)\right\rangle+\nu k^{2}\left\langle\hat{u}_{\beta}^{*}(\boldsymbol{q}, s) \hat{u}_{\alpha}(\boldsymbol{k}, t)\right\rangle\right] .(6)
\end{aligned}
$$

Here $\Lambda_{\alpha}(\boldsymbol{k}, t)$ is the $\alpha$ component of the sum of the first and second terms on the right hand side of the NavierStokes equations, defined similarly as in the shell model case. Further simplification can be made for the sum of the diagonal parts as

$$
\begin{gathered}
\frac{1}{2} \sum_{\alpha=\varphi, \theta} \int G_{\alpha \alpha}^{(T)}(\boldsymbol{k}, t \mid \boldsymbol{k}, s) \frac{d \Omega_{k}}{4 \pi k^{2}}=\frac{1}{\sigma(k)^{2} T}\left[\nu k^{2} E(k ; t-s)\right. \\
\left.-\frac{1}{2}(\mathcal{T}(k ; t, s)+\mathcal{T}(k ; s, t))\right] \frac{1}{4 \pi k^{2}},
\end{gathered}
$$

where the integral is over the surface of the sphere of radius $k$. Here we assume isotropy of the 2ndorder tensors $\left\langle\hat{u}_{c}^{*}(\boldsymbol{k}, s) \hat{u}_{a}(\boldsymbol{k}, t)\right\rangle$ and $\left\langle\Lambda_{c}^{*}(\boldsymbol{k}, s) \hat{u}_{a}(\boldsymbol{k}, t)\right\rangle$ with respect to $\boldsymbol{k}$ and use the two-time energy spectrum function $E(k ; t-s)=\frac{1}{2} \int \sum_{a=1}^{3}\left\langle\hat{u}_{a}^{*}(\boldsymbol{k}, s) \hat{u}_{a}(\boldsymbol{k}, t)\right\rangle d \Omega_{k}$ and the two-time energy transfer function $\mathcal{T}(k ; t, s)=$ $\frac{1}{2} \int \sum_{a=1}^{3}\left\langle\Lambda_{a}^{*}(\boldsymbol{k}, s) \hat{u}_{a}(\boldsymbol{k}, t)\right\rangle d \Omega_{k}$.

The FRRs of the diagonal parts, Eqs. (5) and (17), have an interesting structure: deviation from the proportional 
relation between the linear response function and the auto-correlation function is ascribed to the correlations between the nonlinear term and the velocity, which is the nonlinear energy transfer for the equal-time case. This suggests that the energy transfer between scales, or the energy cascade, causes the deviation. We observe also that the condition $H_{j j}^{(T)}(0)=1$ is satisfied if the squared modulus of each mode is in a statistically steady state.

Numerical analysis of the shell model The shell model Eq. (11) with the total 19 shell variables $(N=18)$ is numerically solved to check whether or not the expression of the response function $H_{j j}^{(T)}$ with the noise (the right hand side of Eq. (5D)) as $T \rightarrow 0$ approaches the one without the noise. We use a forth-order Runge-Kutta scheme with the time step $\Delta t=10^{-4}$. The parameter values are $k_{0}=6.25 \times 10^{-2}, F_{j}=5 \times 10^{-2}(1+i) \delta_{j 0}, \quad \nu=$ $1.66 \times 10^{-5}$, yielding the shell-model analogue of the Taylor-microscale Reynolds number $\operatorname{Re}_{\lambda}=3.9 \times 10^{6}$ and of the large-scale turnover time $\tau_{L}=0.60=6000 \Delta t$ [25].

We use a common numerical method to directly measure $G_{j j}^{(T)}(t-s)$, without using the probe force, by following difference between a pair of orbits, $\Delta u_{j}(t)$ with one orbit being slightly displaced from the other at time $s$ by $\Delta u_{j}(s)$. This yields $G_{j j}^{(T)}(t-s)=\left\langle\Delta u_{j}(t)\right\rangle / \Delta u_{j}(s)$ $[1,12$. The pair share the same realization of the noise. The value of the past displacement $\Delta u_{j}(s)$, taken here to be real, is set to five percents of the standard deviation of the real part of $u_{j}$. We start with twenty different random initial conditions, where all the real and imaginary parts of $u_{j}$ are set by uniformly distributed random variable between -1 and 1 . We first discard data upto $t_{1}=3.3 \times 10^{4} \tau_{L}$ as initial transients and measure the correlation functions and the response functions from $t_{1}$ to $t=8.3 \times 10^{5} \tau_{L}$. The number of samples in calculation of $G_{j j}^{(T)}$ is $5 \times 10^{5}$. For the variance of the noise, we here report the result with a simple choice $\sigma_{j}^{2}=1$. We test two other $k$-dependent settings, $\sigma_{j}^{2}=\nu k_{j}^{2}, \nu k_{j}^{4 / 3}$, and find that the case $\sigma_{j}^{2}=1$ yields the fastest approach to the noiseless system at temperature $T=10^{-4}$ as shown below. Here we do not intend to study the system by varying the power of the wavenumber in the variance in the framework of the renormalization-group analysis of turbulence [30 32]. The expression $H_{j j}^{(T)}$ is obtained by calculating separately the three correlation functions on the right hand side of Eq. (5).

In Fig. 1 we show the time-averaged second-order moment of $\left|u_{j}\right|$ exhibiting the inertial-range scaling $k_{j}^{-\zeta_{2}}\left(\zeta_{2}=0.709\right)$ 25] and the averaged energy flux function $\left\langle\Pi\left(k_{j}\right)\right\rangle=\left\langle\sum_{l=j+1}^{N} \operatorname{Re}\left[\left(\Lambda_{l}-F_{l}\right) u_{l}^{*}\right]\right\rangle$ for various temperatures, indicating that the basic statistics of the Langevin shell model, as $T \rightarrow 0$, become closer to those of the noiseless shell model $(T=0)$. For the lowest temperature $T=10^{-4}$, shown in Fig. 1. now let us demonstrate that the expression of the response function $H_{j j}^{(T)}$ agrees both with the directly measured response

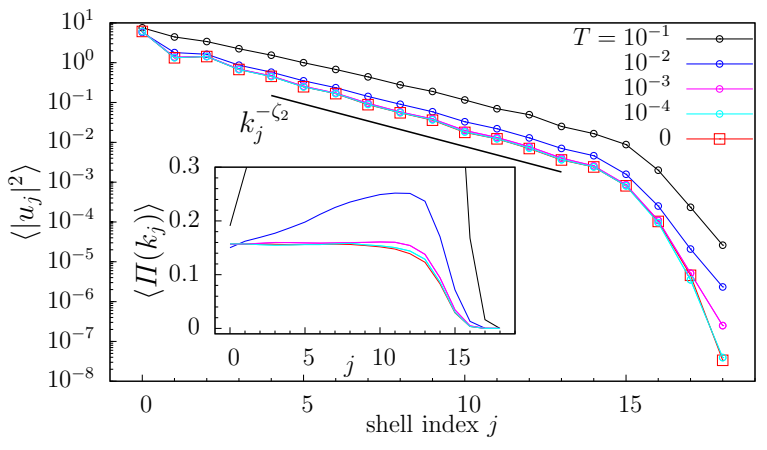

FIG. 1: (Color online) Second-order moment of the absolute value of the shell-model variable $u_{j}(t)$ with and without the Gaussian white noise, as a function of the shell index. Here $\sigma_{j}^{2}=1$ in Eq. (2). Inset: the energy flux function $\Pi\left(k_{j}\right)$ showing that the constant-energy-flux structure is preserved for $T \leq 10^{-3}$.

function $G_{j j}^{(T)}$ and with that of the noiseless case $G_{j j}^{(0)}$ in Fig. 2] [27]. Firstly, we observe that $G_{j j}^{(T)}$ approaches $G_{j j}^{(0)}$ as decreasing $T$, which is displayed in the inset of Fig. 2. With $T=10^{-4}$ the difference between $G_{j j}^{(T)}$ and $G_{j j}^{(0)}$ is less than a few percents for all the shell indices. Secondly, the autocorrelation function $C_{j j}^{(T)}$ also approaches $C_{j j}^{(0)}$ for all the indices as well. As observed in [12], for any index $j, G_{j j}^{(0)}$ is not proportional to $C_{j j}^{(0)}$ (only the case for $j=12$ is presented in Fig. (2). Lastly, $H_{j j}^{(T)}$ agrees with $G_{j j}^{(0)}$ within a few percents for the shells $9 \leq j \leq 18$ covering from the middle of the inertial range to the end of the dissipation range. Four of these shells, $9 \leq j \leq 12$, are presented in Fig. 2. Note also that $H_{j j}^{(T)}$ agrees with $G_{j j}^{(T)}$ for the higher temperature cases, even though $G_{j j}^{(T)}$ is distinctly different from $G_{j j}^{(0)}$ as shown in the inset of Fig. 2 ,

For the shells $0 \leq j \leq 8$, a discrepancy is observed for $T=10^{-4}$ as displayed in Fig. 3(a). The half widths of the errorbars of $G_{j j}^{(0)}$ in Fig. [3(a) correspond to the standard deviations among the $5 \times 10^{5}$ samples of $G_{j j}^{(0)}$. Now we argue that this discrepancy between $H_{j j}^{(T)}$ and $G_{j j}^{(0)}$ observed for small shell indices is not physical but numerical. This is caused by cancellation of the significant digits in the sum of the last two terms in Eq. (5D). Empirically, if the sum of the two terms $\operatorname{Re}\left[\left\langle\Lambda_{j}^{*}(t) u_{j}(s)\right\rangle\right]$ and $\operatorname{Re}\left[\left\langle\Lambda_{j}^{*}(s) u_{j}(t)\right\rangle\right]$, having opposite signs, loses more than two significant digits, agreement between $H_{j j}^{(T)}$ and $G_{j j}^{(0)}$ is lost as indicated in Fig. 3(b). It is difficult to obtain third-order correlation functions of $u_{j}$ like $\left\langle\Lambda_{j}^{*}(t) u_{j}(s)\right\rangle$ with three or more digit accuracy. In fact, for small $j$ 's, the energy transfer correlations $\left\langle\Lambda_{j}^{*}(t) u_{j}(s)\right\rangle$ and $\left\langle\Lambda_{j}^{*}(s) u_{j}(t)\right\rangle$ become increasingly symmetric with the horizontal axis except around the origin $t=s$ as shown in Fig. 3 (c), being a structure likely in common to the 
Navier-Stokes case. Nevertheless, this symmetry is weakened with a larger temperature $T=10^{-3}$ (since the noise breaks the time-reflection symmetry) and a better agreement is obtained for $j=4$ as in the inset of Fig. 3(a).

Concluding remarks We derived formally the FRR of a statistically steady turbulent state of the shell model and the incompressible Navier-Stokes equations with the Langevin noise by using the method of [13, 14]. For the shell model case, as decreasing the amplitude of the noise, we demonstrated numerically that for the intermediate and small scales the derived FRR expression of the linear response function is indeed consistent with that of the noiseless shell model. We consider the discrepancy observed in the large scales as caused by the limited accuracy of the statistical quantities. Our conclusion is that for all the shells the FRR, Eq. (5), as $T \rightarrow 0$, converges to the response function of the shell model without the noise. For the Navier-Stokes case, our preliminary numerical result on two-dimensional inverse-cascade turbulence with a feasible averaging time indicates that Eq. (7) for small $T$ is a good approximation of the response function of the noiseless system. We encounter numerical difficulties similar to the shell model case. A numerical assessment of the FRR, Eq. (7), will be re- ported elsewhere. Regarding the intermittency, it does affect each term in the FRR. However, the FRR on the whole remains unaffected. This suggests that the FRR may be a bridge relation of the intermittency or dynamic multiscaling (see, e.g., [28, 29]) among the 2nd-order, 3rd-order correlation functions and the response function if the intermittency is not susceptible to the small Langevin noise. With this bridge relation, however, the wavenumber dependence of the integral time of the response function may not be described by the dominant multiscaling exponents of the 3rd-order correlation functions, since the cancellation occurs as seen in Fig[3(c). Future research directions to take further advantage of the vanishing noise can be to develop a spectral closure approximation with the FRR response function obtained here and to consider saddle-point solutions (instantons) in the integral Eq. (3) as in [22, 33], which may yield an interesting dynamical approach to turbulence.

Acknowledgments We acknowledge delightful discussions with So Kitsunezaki and Shin-ichi Sasa and the support by Grants-in-Aid for Scientific Research (C) No. 21540388 and (C) No. 24540404 from JSPS. A part of the numerical calculation was done on the HPC facilities at RIMS, Kyoto University.
[1] U.M.B. Marconi, A. Puglisi, L. Rondoni, A. Vulpiani, Phys. Rep. 461, 111 (2008).

[2] R.H. Kraichnan, J. Fluid Mech., 5, 497 (1959).

[3] G.L. Eyink and U. Frisch, "Robert H. Kraichnan" in $A$ Voyage Through Turbulence, P.A. Davidson et al. (eds.), Cambridge University Press (2011).

[4] U. Frisch, Turbulence - Legacy of A.N.Kolmogorov, Cambridge Univ. Press (1996).

[5] G. Gallavotti, Foundations of Fluid Mechanics, Springer (2005).

[6] Y. Kaneda, J. Fluid Mech., 107, 131 (1981).

[7] S. Kida and S. Goto, J. Fluid Mech., 345, 307 (1997).

[8] M. Carini and M. Quadrio, Phys. Rev. E 82, 066301 (2010).

[9] E.B. Gledzer, Sov. Phys. Dokl., 18, 216 (1973).

[10] K. Ohkitani and M. Yamada, Prog. Theo. Phys. 89, 329 (1989).

[11] L. Biferale, Ann. Rev. Fluid Mech. 35, 441 (2003).

[12] L. Biferale, I. Daumont, G. Lacorata, and A. Vulpiani, Phys. Rev. E 65, 016302 (2001).

[13] T. Harada and S.-i. Sasa, Phys. Rev. Lett. 95, 130602 (2005).

[14] T. Harada and S.-i. Sasa, Phys. Rev. E 73, 026131 (2006).

[15] S. Toyabe, H.R. Jiang, T. Nakamura, Y. Murayama, and M. Sano, Phys. Rev. E 75, 011122 (2007).

[16] H. Teramoto and S.-i. Sasa, Phys. Rev. E 72, 060102 (2005).

[17] S-H. Chong, M. Otsuki, and H. Hayakawa, Phys. Rev. E 81, 041130 (2010).

[18] H. Hayakawa and M. Otsuki, Phys. Rev. E 88, 032117 (2013).

[19] U. Seifert, Rep. Prog. Phys. 75, 126001 (2012).

[20] M.I. Freidlin and A.D. Wentzell, Random perturbations

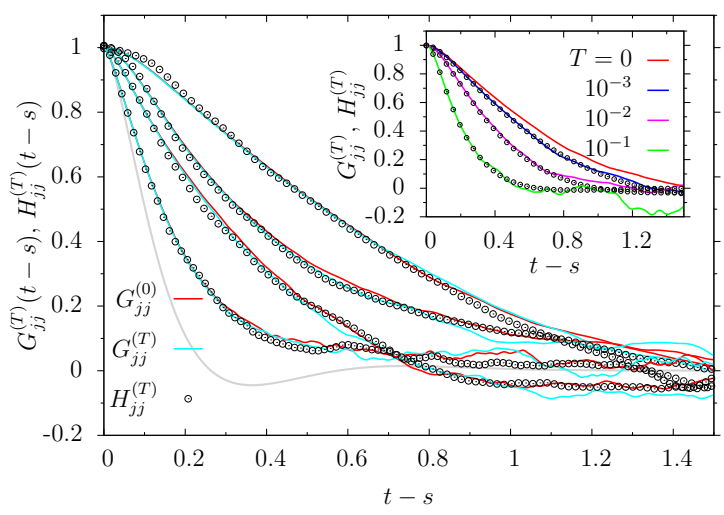

FIG. 2: (Color online) Directly calculated response function of the shell model $G_{j j}^{(0)}$ (zero temperature), $G_{j j}^{(T)}$ with $T=10^{-4}$, and the FRR expression of the response function $H_{j j}^{(T)}$ with $T=10^{-4}$, the right hand side of Eq. (5), for the shell indices $j=9,10,11$, and 12 (from top to bottom). The gray curve is $C_{j j}^{(0)}(t-s) / C_{j j}^{(0)}(0)$ for $j=12$. Inset: approach of $G_{j j}^{(T)}$ to $G_{j j}^{(0)}$ as $T \rightarrow 0$ for $j=9$, plotted with $H_{j j}^{(T)}$.

of dynamical systems, Springer (2012).

[21] F. Flandoli, Random perturbation of PDEs and Fluid dynamic models, Lec. Notes Math., 2015, (2011).

[22] J. Kurchan, J. Stat. Phys., 128, 1307 (2007).

[23] V.S. L'vov, E. Podivilov, A. Pomyalov, I. Procaccia, and D. Vandembroucq, Phys. Rev. E 55, 1811 (1998).

[24] R. Graham, Z. Physik B 26, 281 (1977). 

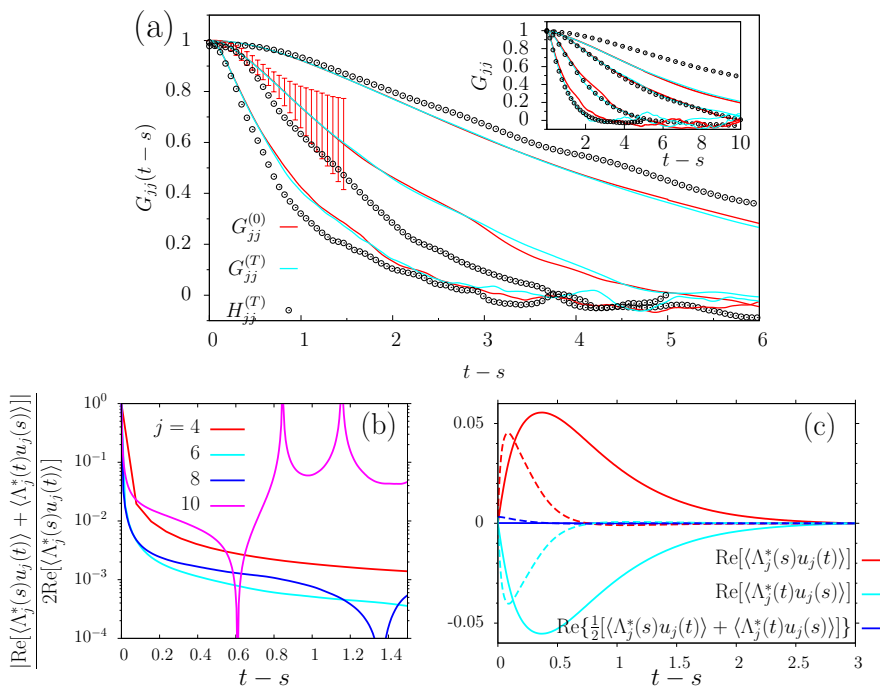

FIG. 3: (Color online) Numerical difficulty of the FRR. (a) Discrepancy between $H_{j j}^{(T)}$ and $G_{j j}^{(0)}$ for $j=4,6$, and 8 (from top to bottom) with $T=10^{-4}$; The errorbars of $G_{j j}^{(0)}$ are shown for the index $j=6$ up to $t-s=1.5$. Inset: same as the outset but for $j=2,4,6$, and 8 with $T=10^{-3}$. (b) Cancellation between the real parts of $\left\langle\Lambda_{j}^{*}(s) u_{j}(t)\right\rangle$ and $\left\langle\Lambda_{j}^{*}(t) u_{j}(s)\right\rangle$ with $T=10^{-4}$. (c) Symmetry of the real parts of $\left\langle\Lambda_{j}^{*}(s) u_{j}(t)\right\rangle$ and $\left\langle\Lambda_{j}^{*}(t) u_{j}(s)\right\rangle$ for $j=8$ (solid) and 11 (dashed) with $T=10^{-4}$.
[25] D. Mitra and R. Pandit, Phys. Rev. Lett. 93, 024501 (2004). In this Ref. the Reynolds number $\operatorname{Re}_{\lambda}=u_{\mathrm{rms}} \lambda / \nu$ is defined with the Taylor microscale $\lambda=\left[\left(\sum_{j} k_{j}^{-1}\left|u_{j}\right|^{2}\right) /\left(\sum_{j} k_{j}\left|u_{j}\right|^{2}\right)\right]^{1 / 2}$ and the root-meansquare velocity $u_{\mathrm{rms}}=\left[2 \sum_{j} k_{j}^{-1}\left|u_{j}\right|^{2} /\left(2 \pi k_{0}\right)\right]^{1 / 2}$. The large-scale turnover time is $\tau_{L}=L / u_{\mathrm{rms}}$, where the integral scale is $L=\left(\sum_{j} k_{j}^{-2}\left|u_{j}\right|^{2}\right) /\left(\sum_{j} k_{j}^{-1}\left|u_{j}\right|^{2}\right)$.

[26] In the decomposition the orthonomal vectors $\boldsymbol{e}_{\varphi}$ and $\boldsymbol{e}_{\theta}$ are associated with respectively the azimuthal angle $(0 \leq$ $\varphi \leq 2 \pi)$ and the polar angle $(0 \leq \theta \leq \pi$, defined as the angle from the positive part of the $k_{z}$ axis) for a given $\boldsymbol{k}$ in the spherical coordinates of the three dimensional wavenumber space.

[27] Hereafter, we consider only the real parts of $G_{j j}^{(T)}(t-s)$ and $H_{j j}^{(T)}(t-s)$ since the absolute values of their imaginary parts are smaller than 0.12 for $0 \leq t-s \leq 1.5$ for the shells shown in Fig. 2

[28] L. Biferale, E. Calzavarini and F. Toschi, Phys. Fluids 23, 085107 (2011).

[29] S.S. Ray, D. Mitra, P. Perlekar and R. Pandit, Phys. Rev. Lett. 107, 184503 (2011).

[30] A. Sain, Manu and R. Pandit, Phys. Rev. Lett. 81, 4377 (1998).

[31] L. Biferale, A.S. Lanotte and F. Toschi, Phys. Rev. Lett. 92, 094503 (2004).

[32] A. Mazzino, P. Muratore-Ginanneschi and S. Musacchio, Phys. Rev. Lett. 99, 144502 (2007).

[33] A. Baule, H. Touchette, and E.G.D. Cohen, Nonlinearity, 24, 351 (2011). 